\title{
Innovación socialk estrategias urbanas en un contexto de cambio. El caso de la ciudad de Valencia
}

\section{Julia Salom-Carrasco, María-Dolores Pitarch-Garrido, Ana Sales-Ten}

RESUMEN: El objetivo de este artículo es presentar los resultados de un análisis y cartografía de las iniciativas de innovación social que han surgido en la ciudad de Valencia en los últimos años. Mediante trabajo de campo y técnicas fundamentalmente cualitativas (FocusGroup, entrevistas, cuestionarios a informadores cualificados), se han recopilado y analizado las iniciativas de este tipo que se encuentran actualmente activas, analizando su tipología, cronología y localización geográfica, con el fin de determinar las variables que han condicionado su nacimiento y, en particular, su posible vinculación con las características de los barrios en que se han originado (problemática social, estructura sociodemográfica, tradición participativa, redes y capital social existente, etc.). Los resultados muestran la gran diversidad temática existente, así como su vinculación con las demandas percibidas por la población y su vinculación a barrios con población joven y áreas consolidadas con tradición participativa, como Ciutat Vella, Benimaclet y Marítim.

PALABRAS CLAVE: Innovación social, crisis urbana, barrios, Valencia.

CLAVES ECONLIT: L31, O35, R11, R23, 031.

Cómo citar este artículo / How to cite this article: SALOM-CARRASCO, J., PITARCH-GARRIDO, M.D. \& SALES-TEN, A. (2017): "Innovación social: estrategias urbanas en un contexto de cambio. El caso de la ciudad de Valencia", CIRIEC-España, Revista de Economía Pública, Social y Cooperativa, 91, 31-58.

Correspondencia: Universitat de València, Instituto Interuniversitario de Desarrollo Local,

Departamento de Geografía, C/. Serpis, 29 - 46022, Valencia, España. E-mail de contacto: Julia.Salom@uv.es 


\section{EXPANDED ABSTRACT}

\section{Social innovation: urban strategies in a context of change. The case of the city of Valencia}

In recent years, the concept of social innovation has been receiving a lot of attention from both social scientists and decisions makers. The most widespread idea among the latter is that it can be a viable alternative for solving social problems, because it seems that they can offer solutions to problems that neither government policy nor the market have been able to solve. Despite this, research on social innovation in academia is still scarce and it largely focusses on the analysis of case studies. Besides having a definition, it is essential to know the factors that brought about its materialization, as well as the process leading to its consolidation and spread.

With a view to identifying, making visible and disseminating good practice, and also reflecting on the concept, the project of the social innovation map for the city of Valencia was raised, as a result of the collaboration between the University of Valencia and Las Naves Foundation of Valencia City Council. The city of Valencia is one of the large Spanish cities that has suffered most intensely the effects of the recent economic crisis, due to the immense importance that the real estate sector and lesser technological activities had acquired in previous years. Economic regression has had important social effects, giving rise to an extremely augmented and increasingly polarised urban space. In this context, the aim of the project is to learn about the social innovation initiatives that exist in the city of Valencia, their type and origin, as well as to understand and analyse the specific social scenario in which they are developing.

\section{METHODOLOGY}

Qualitative and quantitative techniques have been applied alongside each other in the study, working mainly on two lines of research.

The first focused on analysing the concept of innovation, with the aim of defining common criteria which would allow us to select and characterise the initiatives. The techniques applied to this end have been: (1) documentary research, (2) conducting an online consultative survey of 51 university professionals from different disciplines on the most important aspects of social innovation, and (3) holding a focus group with experts on the matter from both academic and professional spheres.

The second line of work consisted in gathering and analysing case studies in the city of Valencia. Given that there was no existing information, local calls for projects were consulted, as well as trawling through social networks, webs and various documentary sources. This initial enquiry enabled us to register a total of 112 initiatives, which, after filtering these, were reduced to 79 , classified in seven 
categories depending on their main objective. Their geographical location in the urban environment allowed us to deduce some important aspects of the environment in which they arose.

\section{RESULTS}

\section{Contributions to the definition and use of the concept}

Social innovation is a term that has many and varied interpretations. As common aspects, the experts taking part in the focus-group pointed to the following elements:

- It is preferable to talk about sociocultural innovation, because there is not a single social process that is not at the same time cultural; if there is no cultural change, there can hardly be any social innovation.

- Social innovation requires creativity, which brings with it the following elements: originality, adaptation, a future-oriented approach and the ability to solve social problems. However, the criterion of originality opens up a debate between, on the one hand, opinions based on the definition of social innovation according to Stanford Social Innovation, and on the other, those who support the definition of María Elisa Bernal, based on the experiences of UNECLAC. This latter proposes that social innovation involves new ways of doing traditional jobs, and that these jobs must be replicable in different contexts, so that the novelty is not as important as it is for the experts at the University of Stanford.

- Another point of agreement is the role of the Administration. Although this acts as a source of different processes for social innovation, there are administrative structures which often inhibit initiatives being taken, and which still continue to promote standardised and unidirectional responses to complex multi-dimensional problems. There is a lack of dialogue, collaboration, synergy and confusion among the different agents promoting Social Innovation. Innovation in the public sphere is too limited to certain environments and restricted by its own economic, organisational and relational structure.

Moreover, the results of the surveys of university teachers show us the conceptual differences arising from the various disciplinary perspectives. So while economists and sociologists give great importance to social challenges, geographers, historians and healthcare professionals consider aspects relating to community development important. However, within these two groups there are also differences: On the one hand, while geographers and historians attach no importance to putting the idea into practice, this for doctors is one of the most significant aspects. On the other hand, economists highlight novelty, whilst sociologists do not.

\section{Type of initiatives found, geographical location and relations with districts}

The 79 initiatives identified have been grouped into seven categories organised according to the aim of the innovation in each of them, the category 'Social dynamics and inclusion' being the one that groups the largest number of initiatives ( $24 \%$ of the total), followed by the one called 'Exchange and collaborative economy networks' (19\%). In all of them the Third Sector predominates over other organisational forms such as self-organisation and the private and, above all, public sectors. The date initiatives started shows a significant upturn as of 2013 , which can be related to the socio-political context at the time. 
Concerning the factors that propitiated their appearance, the results show that this is closely linked with the socio-demographic, economic and territorial conditions of the environment in which they appear. The innovative initiatives identified are concentrated in three main urban areas: 1) the district of Ciutat Vella and the neighbouring district of El Botànic; 2) Benimaclet, and the neighbouring districts of Algirós and Rascanya, and 3) Els Poblats Marítim.

Although it is evident that we find ourselves facing complex social processes in which different variables intervene, and whose interpretation cannot be made except after a detailed qualitative analysis, this location points to the importance of the following variables:

a) Firstly, the existence of initiatives seems to be related to the income level of the population. Literature on social innovation considers that this arises as a means of combatting social exclusion and enhancing the quality of life; this would explain the practical absence of these types of projects in districts inhabited by populations with a high income and the absence of conflicts relating to multiculturalism.

b) However, the income variable is an insufficient explanation, since the most active hubs of social innovation do not necessarily match with the worst situation. Therefore, a second explanatory variable points to the link with social and citizen movements, in our case with the "indignant" movement, which had particular impact on the city of Valencia. The date on which they arose indicates this (65\% of the ones detected grew up between 2014 and 2016) and their intensity in the districts which presented a larger percentage of votes for parties related to this movement in the 2015 local elections.

c) But perhaps the most important variable is the social and associative fabric, the networks and the social capital consolidated over time. Thus, the three main innovation nuclei coincide with areas with a long tradition of formal or informal sociability, important associative hubs, linked to a powerful neighbourhood movement, an exceptionally dense festive framework, and different types of recreational or cultural associations; social networks which have been set up in response to a situation of prolonged marginalization and to territorial and social conflicts that have affected them.

\section{CONCLUSIONS}

The results of the research conducted enables us to conclude that, although there is a coincidence between the various specialists when pointing to the main aspects of social innovation as cultural change, creativity, adaptation, looking to the future and problem-solving ability, there are also marked differences arising from the different level of familiarity with the concept, and diverse disciplinary perspectives. In this respect, a significant point of agreement is the limited role of the Administration which, although it occasionally acts as a source of different social innovation processes, presents administrative structures which inhibit taking initiatives and continue to promote standardized and unidirectional responses to complex and multi-dimensional problems. 
Moreover, the empirical analysis has enabled us to point to the importance of geographical conditions (socio-demographical, economic and territorial) in the emergence of these initiatives. Among these conditions, the poor socio-economic situation must be highlighted, but also, and decisively, the link to social and citizen movements, and the existence of social networks and the social capital consolidated over time.

KEYWORDS: Social innovation, Urban crisis, Neighbourhoods, Valencia.

\section{Introducción: el concepto de innovación social y su relación con el desarrollo}

En los últimos años, el concepto de innovación social está recibiendo gran atención por parte tanto de los científicos sociales como de los tomadores de decisiones. La idea más extendida entre estos últimos es que puede ser unaalternativa viable para la solución de problemas sociales, pues parece que este tipo de iniciativas son capaces de ofrecer soluciones a problemas que ni la política del gobierno ni el mercado han sido capaces de solventar (Choiy Majumdar, 2015). La innovación social ha sido, y es, objeto de relevancia política en las agendas de Estados Unidos y de la Unión Europea. En el primer caso, en 2009 la administración Obama crea la Oficina de la Innovación Social y de la Participación Ciudadana $^{1}$, dependiente directamente de la Casa Blanca, que nace con el fin de apoyar el desarrollo de ideas innovadoras que se dirijan a fines sociales. Surge en particular como respuesta al enorme potencial de desarrollo y crecimiento de las organizaciones sin ánimo de lucro con fines sociales que, a pesar de los demostrados beneficios que suponen en un país como Estados Unidos (en 2012 empleaban a 13,5 millones de trabajadores y generaban el 5,5\% del PIB), se enfrentan a la escasez de recursos y a la dispersión de los esfuerzos. Entre las políticas y programas desarrollados hasta la fecha, destaca la Fundación para la Innovación Social2 ${ }^{2}$, creada como entidad de colaboración público-privada con el objetivo de coordinar los nuevos enfoques vinculados a las iniciativas de innovación social con las necesidades sociales, favorecer la financiación privada (filantropía) y pública de dichas iniciativas, y realizar la evaluación de los resultados a partir de evidencias de éxito.

El concepto de innovación social que utiliza la administración estadounidense está basado en el acuñado por la Stanford Social Innovation Review, cuyos editores en 2003 la definen como el proceso de inventar, asegurar apoyo e implementar soluciones novedosas a necesidades y problemas sociales. Sin embargo, el incremento de este tipo de iniciativas en los últimos veinte años ha exigido la

1.- Office of Social Innovation and Civic Participation: https://obamawhitehouse.archives.gov/administration/eop/sicp

2.- Social Innovation Fund: https://www.nationalservice.gov/programs/social-innovation-fund 
ampliación de esta definición para incluir nuevas realidades y diferenciarla de otros términos como el de empresa social. En consecuencia, en el año 2008 se redefinió el término como "una solución nueva a un problema social que es más efectiva, eficiente, sostenible o justa que las soluciones existentes y para el cual el valor creado se orienta principalmente a la sociedad en su conjunto más que a los particulares" (Phills, Deiglmeier y Miller, 2008, p. 35). El objetivo tanto metodológico como político que se plantea en relación con este concepto es conocer cómo el libre flujo de ideas, valores, relaciones y dinero entre los distintos sectores está fomentando los procesos de innovación social contemporáneos capaces de poner en marcha soluciones nuevas y duraderas a los problemas sociales más acuciantes de nuestro tiempo. Por ello, el principal objetivo de la administración norteamericana es conocer y potenciar las buenas prácticas, es decir, las experiencias de éxito.

En la Unión Europea el término innovación social también ha tenido un gran seguimiento e impacto. EIReglamento para el Programa de la UE para el Empleo y la Innovación Social (EaSI) de diciembre de 2013, indica, siguiendo las iniciativas de Europa 2020, que la innovación social es una herramienta para "abordar los desafíos sociales que plantea el envejecimiento de la población, la pobreza, el desempleo, los cambios en las costumbres laborales y personales, y las expectativas de los ciudadanos con respecto a la justicia social, la educación y la atención sanitaria." Teniendo esto en cuenta, las acciones que se llevan a cabo para potenciar la innovación social son objeto de financiación por parte de la UE, en particular en el caso de las asociaciones transnacionales y la creación de redes entre los agentes públicos, privados y del tercer sector. Se indica como objetivo sustancial la identificación de soluciones innovadoras, que funcionen; y su aplicación práctica a partir de la implementación de políticas sociales. En el artículo 2, ap. 5 se definen las innovaciones sociales como aquéllas "que sean sociales tanto por sus fines como por sus medios, y en particular las que se refieran al desarrollo y la puesta en práctica de nuevas ideas (relacionadas con productos, servicios y modelos) que, simultáneamente, satisfagan necesidades sociales, beneficiando de esta forma a la sociedad y reforzando su capacidad de actuación". Además, en el apartado 6 de este mismo artículo se define experimentación en política social como aquellas "intervenciones de política que aportan respuestas innovadoras a las necesidades sociales, aplicadas a pequeña escala y en condiciones que permiten medir su impacto, antes de su repetición a mayor escala si los resultados se demuestran convincentes."3

A pesar del gran interés despertado entre los políticos en los últimos años, la investigación sobre innovación social en la academia es todavía escasa (Choi y Majumdar, 2015, Hernández-Ascanio, Tirado-Valencia y Ariza-Montes, 2016). La mayor parte de los estudios son análisis de casos prácticos que pueden -o no- ser definidos como de innovación social. La Unión Europea ha financiado algunos proyectos de investigación sobre el tema, al igual que algunas universidades, pero el concepto todavía crea controversia. Sin embargo, la definición de un marco conceptual resulta clave en términos económicos en general y en las estrategias de desarrollo social en particular. La falta de unanimidad en el significado del concepto es reflejo de la diversidad de contextos en los que se desarrollan estas iniciativas. Se podría decir que la innovación social es intersectorial y multidisciplinar, pero tam- 
bién diferente según el contexto territorial en el que se desarrolla. Por todo ello, desde distintos campos científicos se han elaboradodiferentes conceptualizaciones.

Entidades como la citada Universidad de Stanford (EE.UU), la Young Foundation en Reino Unido, la Comisión Económica para América Latina y el Caribe (CEPAL) y la OECD(Manual de Oslo) ${ }^{4}$, entre otras, dan sus propias definiciones. Cabe destacar que una de las diferencias más importantes entre ellas es en qué aspecto ponen el acento. Mientras que Stanford destaca la aplicación práctica dela innovación social más que una definición teórica del concepto, la Comisión Europea, como hemos señalado, se preocupa de que este tipo de iniciativas solvente los grandes retos de la sociedad. Por su parte, la OCDE (2010) destaca la participación de la comunidad y de los beneficiarios; y autores como Moulaert et al. (2005) hacen hincapié en el proceso (que incluye cambios en las dinámicas y relaciones sociales) y, sobre todo, en el contexto, como veremos más adelante.

Mulgan (2007), director de la Young Foundationy seguidor de la línea desarrollada por la Universidad de Stanford, indica que, además de contar con una definición, es fundamental conocer por qué es importante, cómo puede acelerarse, qué actores están implicados, qué resistencias existen, y cuáles son las fases de desarrollo de una innovación social, desde su creación hasta su difusión. Caulier-Grice et al. (2012), en esta misma línea, definen la inovación social como "nuevas soluciones (productos, servicios, modelos, mercados, procesos, etc.) que simultáneamente responden a una necesidad social (más efectivamente que las soluciones existentes) y se orientan hacia nuevas o mejoradas capacidades y relaciones, y hacia un mejor uso de los bienes y recursos. En otras palabras, las innovaciones sociales son tanto buenas para la sociedad como para mejorar su capacidad de acción" (p. 18).

También Murray, Caulier-Grice y Mulgan (2010), siguen esta línea para la definición de innovación social en su Open Book of Social Innovation publicado por la Young Foundation, indicando que ésta se puede definir como el conjunto de nuevas ideas (productos, servicios y modelos) que satisfacen las necesidades sociales y simultáneamente crean nuevas relaciones sociales y redes de colaboración. Estos autores, más interesados por las prácticas innovadoras que por la definición del concepto, señalan que la razón por la que este tipo de iniciativas ha crecido tanto en la última década es porque las estructuras y políticas existentes se han demostrado ineficaces para enfrentar los retos más importantes de nuestra era, como el cambio climático, las enfermedades crónicas y las epidemias, y la brecha cada vez más amplia de la desigualdad. El mercado tampoco ha desarrollado los mecanismos para solucionar estos problemas, por lo que allí donde el mercado falla, el Estado o la sociedad civil deben actuar. En este momento histórico, es la sociedad la que ha tomado la iniciativa, pues las soluciones de la política son, según estos autores, antiguas (se limitan a repetir modelos obsoletos) y poco realistas. Frente a esto, la sociedad está normalmente falta de capital, conocimientos o habilidades y

4.- La OCDE en el llamado Manual de Oslo, habla fundamentalemnte de innovación en servicios. Primera edición en 1996. Última edición (remodelada) en 2006 ( $3^{a}$ ed.). Ver OECD/European Communities (2005) y Manual de Oslo: http://www.oecd-ilibrary.org/science-andtechnology/manual-de-oslo_9789264065659-es;jsessionid=e2tcaq6h78qqq.x-oecd-live-02 
recursos para poder ampliar la escala de impacto de las nuevas ideas, por lo que la colaboración público-privada resulta imprescindible. Está demostrado que los nuevos paradigmas tienden a desarrollarse en aquellas áreas en donde las instituciones son más receptivas y apoyan el desarrollo de ideas innovadoras, normalmente en el margen de la estructura administrativa central. Los autores califican las iniciativas de innovación social como una "economía social emergente" (p. 4) con unas características propias que incluyen: un uso intensivo de las redes de distribución para sostener y gestionar las relaciones (internet), límites difusos entre la producción y el consumo, énfasis en la colaboración y las interacciones, más que en el consumo, y, por último, un fuerte peso de los valores y misiones de cada una de las iniciativas sociales, basadas en el creciente valor de la dimensión humana ("poner a las personas primero").

Un elemento de importancia, en particular para los geógrafos, es el impacto que la innovación social tiene a nivel local o territorial. En esta línea, Moulaert (2007, 2010 y 2013) destaca el peso de la innovación como motor del desarrollo social de las ciudades europeas por su papel en la inclusión de grupos sociales desfavorecidos y tradicionalmente sin voz política. Moulaert et al. (2013) han definido recientemente la innovación social como un conjunto de soluciones puestas en marcha por los ciudadanos para satisfacer sus necesidades sociales de manera diferente a las propuestas por el mercado o las instituciones públicas, buscando el desarrollo local sostenible y el control de su propio crecimiento por parte de la población. Relacionan la innovación social con la dinámica de empoderamiento, dando así un carácter político a los movimientos sociales y las iniciativas de gobernabilidad de abajo hacia arriba. Esta definición abarca una gran diversidad de acciones o prácticas, desde los microcréditos a las monedas sociales, pero siempre con un positivo impacto territorial (local, comunidades y barrios) y de cambio social. Según Moulaert et al. (2005), la innovación social incorpora explícitamente un posicionamiento ético sobre la justicia social y los valores de la convivencia humana, lo cual resulta una aportación de gran importancia, a pesar de la controversia que pueda suscitar. En definitiva, la innovación social tiene mucho que ver con la inclusión social, las relaciones de poder y la lucha a nivel local contra las fuerzas dominantes, excluyentes, del mercado.

En relación con la línea argumental anterior, se encuentra la desarrollada por la CEPALy financiada por la Fundación W.K. Kellogg, que analiza este tipo de innovaciones en América Latina, territorio en el que, quizá más que en otros lugares, la mayor parte de las iniciativas no son suficientemente visibles. Las acciones estudiadas se dirigen específicamente a temas de gran impacto social, en áreas como la salud, la educación, la generación y mejora de los ingresos, y la atención a los jóvenes y las mujeres en riesgo de exclusión. Cabe señalar que, a partir del estudio de las iniciativas de innovación social en América Latina, la CEPAL considera que el objetivo principal de las mismas es solucionar problemas sociales concretos, por lo que la replicabilidad, un elemento clave en otras definiciones, no se considera un requisito exigible, ya que"que la innovación consiga mejorar la respuesta a esas necesidades es independiente de su réplica en otras esferas" (Rodríguez- Herrera y Alvarado, 2008, p.22). Sin embargo, es cierto que muchas de las iniciativas desarrolladas en este campo son copias mejoradas 0 adaptadas de otras puestas en marcha en otros entornos o en otros temas. Lo que parece ocurrir es, precisamente, una adaptación de las innovaciones originales a las características del terri- 


\section{INNOVACIÓN SOCIAL: ESTRATEGIAS URBANAS EN UN CONTEXTO DE CAMBIO. EL CASO DE LA CIUDAD DE VALENCIA}

torio concreto en el que existe el problema social a resolver. Es, por tanto, una perspectiva muy ligada al desarrollo local y sostenible. Así, Rodríguez-Herrera y Alvarado (2008) señalan que "las innovaciones en el campo social a menudo surgen en condiciones adversas, en entornos en los que el mercado no ha ofrecido alternativas ni el sector público ha respondido a las necesidades y reclamos de la población. Por este motivo, muchas veces su puesta en práctica encuentra obstáculos al intentar trascender el ámbito local en que se originan o multiplicar el número de beneficiarios. Los encargados de formular e implementar las políticas públicas deberían vincularse con estas innovaciones, facilitar su concreción y recoger sus aportes" (p.13). El valor dado a la intervención del sector público para mantener y fomentar la innovación social es fundamental en este enfoque.

El cambio social es el objetivo final de ambos enfoques, por lo que las diferentes formas de entender la innovación social no son, necesariamente, excluyentes. Al contrario, como se ha señalado, aunque se haga diferente énfasis en aspectos específicos, existen muchos factores comunes.

Más allá de las tradiciones epistemológicas, y de forma más pragmática, es posible distinguir dos bloques en la forma de concebir la innovación social (Martínez y Subirats, 2015):

a) De acuerdo con el enfoque económico o de gestión, son los llamados emprendedores sociales los encargados de desarrollar nuevas ideas, productos o servicios con el objetivo de satisfacer necesidades sociales,

b) Desde un enfoque socioecológico, se considera que es la sociedad la que favorece y, en cierta manera, determina la aparición de innovaciones. Se trata de un proceso complejo en el que las innovaciones sociales cambian profundamente las rutinas básicas, los recursos, el empoderamiento y las creencias del sistema social.

Sin embargo, resulta mucho más interesante centrarse no sólo en las características estructurales de este fenómeno, sino en analizar el proceso que da lugar a la aparición de nuevas estructuras colaborativas y de participación, a lo que hay que añadir la capacidad de generar procesos de co-creación y empoderamiento de grupos sociales con una menor capacidad de intervención en la vida económica, social y política, ya que la idea del cambio social subyace en la gran mayoría de este tipo de iniciativas.

A este respecto, desde diferentes paradigmas científicos se apuntan distintas características específicas del proceso:

a) Innovación social como cambio social: Establecimiento y consolidación de nuevas estructuras sociales que supongan cambios en la cultura, normativa o regulación de las actuales (Heiskala, 2007). Supone una renovación de la sociedad en su conjunto, como objetivo final. Para la sociología, esta es la característica más importante de la innovación social.

b) Innovación social como innovación intangible:Las innovaciones dirigidas a unos fines sociales específicos son, pues, acciones y no objetos materiales, aunque puedan servirse de éstos (en especial de las innovaciones tecnológicas). Son ideas puestas en marcha que se manifies- 
tan a partir de la oferta de servicios a la sociedad, incorporan creatividad y novedad. Las ciencias sociales, en particular las ciencias políticas, consideran esta característica como la más definitoria del concepto.

c) Innovación social como innovación que tiene como objetivo crear valor social:supone un cambio social positivo, una mejora de la calidad de vida de las personas y de sus prácticas sociales. Según Choi y Majumdar (2015), el uso explícito del concepto de innovación social como creador de valor es el más adecuado, y ello incluye los niveles de interacción social, práctica social, y también producción de nuevos bienes tangibles, al contrario que en la consideración anterior. Esta característica es la más importante en los enfoques que provienen de la emprendeduría social, el desarrollo territorial y la psicología.

El cambio social ligado a la innovación social, o consecuencia de ésta, es una relación poco explorada por los científicos sociales. Se define cambio social como el proceso en el que emergen nuevas prácticas sociales, que se convierten en socialmente aceptadas y se difunden en la sociedad por procesos de imitación, adaptación y aprendizaje social, condicionados por distintas circunstancias. Finalmente estos cambios son institucionalizados como nuevas prácticas que terminan convirtiéndose en rutinas.

En este sentido, un último concepto relativo a la innovación de gran relevancia, pero poco estudiado en el caso de la innovación social, es el de difusión de las innovaciones. Conocer con qué profundidad y extensión se difunde en la sociedad la innovación, hasta qué punto pone en cuestión las prácticas sociales actuales, y en qué medida y cuándo es institucionalizada, es decir, adaptada para resolver de manera más amplia dichos problemas, son cuestiones que tienen importantes implicaciones en la acción pública (Peraza, Gómez Y Aleixandre, 2016).

En conclusión, el término "innovación social" es uno de esos casos de términos de uso frecuente y falta de consenso en su definición. Se trata de un concepto complejo que incorpora nuevas ideas (productos, servicios o modelos) capaces de responder a necesidades sociales de forma más efectiva que sus alternativas, al mismo tiempo que crea relaciones sociales o formas de colaboración entre las personas que pueden caracterizarse como novedosas. El concepto se encuentra muy relacionado con nociones como solución y transformación.Sin embargo, aunque su definición y desarrollo como categoría analítica precisa de un nuevo marco conceptual, lo que en la práctica adquiere importancia es la identificación de buenas prácticas, lo que se vincula con una valoración (ética) de las mismas, y con su capacidad de influir en la acción por una nueva sociedad, más justa, sostenible y, en definitiva, libre y responsable.

A este respecto, el proyecto "Barrios y Crisis"5 desarrollado por el IGOP (Institut de Govern i Politiques Públiques) ${ }^{6}$ ha sido pionero en el ámbito español, al identificar las iniciativas sociales en

5. - https://barrisicrisi.wordpress.com/equip-de-recerca/ (consultado el 10 de mayo de 2017)

6. - http://igop.uab.cat/es/barrios-y-crisis/ (consultado el 10 de mayo de 2017) 
Cataluña, localizarlas y relacionarlas con los problemas que tratan de resolver. A partir del estudio de las innovaciones sociales y la estructura socioeconómica de las ciudades y barrios, los autores llegan a cuestionar una de las corrientes interpretativas más influyentes en el campo de la innovación social, el llamado «enfoque de base», según la cual la innovación social aparece para paliar el fracaso del mercado y del Estado, es decir, como una forma de lucha contra la exclusión y la vulnerabilidad. Por el contrario, el análisis empírico parece demostrar que, al menos en Catalunya, la innovación social surge en muchas ocasiones en el contexto de espacios de clase media, y asociada a cuestiones que no están relacionadas con la vulnerabilidad, sino con otros temas como la precarización, las formas de vida alternativa, la socialización, etc. La existencia en el territorio de recursos para la acción colectiva, hecho que no debe darse siempre por supuesto, es clave para el desarrollo de las iniciativas de innovación social; $y$, por tanto, es una línea de acción para el apoyo por parte de las políticas sociales (Blanco, Cruz, Martínez, R. y Parés, 2015, López, Priede y del Arco, 2015). Se trata, pues, de un enfoque también relacionado con el desarrollo local, incluso microlocal, como es el barrio, el espacio más cercano, sus características socioculturales y su tradición y/o capacidad asociativa.

En esta línea de identificación, visibilización y difusión de buenas prácticas se plantea el proyecto del mapa de innovación social de la ciudad de Valencia, como una iniciativa de la Cátedra institucional Ciudad de Valencia, fruto de la colaboración entre la Universidad de Valencia y la Fundación Las Naves del Ayuntamiento de Valencia. Esta iniciativa parte de la consideración de que el análisis de las iniciativas identificadas puede aumentar el conocimiento de los procesos y factores que contribuyen a su nacimiento y desarrollo, información útil para mejorar el diseño de políticas de apoyo específicas que contribuyan a la solución de problemas sociales relevantes. En las páginas siguientes se presenta la metodología aplicada, los principales resultados, y la relación que existe entre el nacimiento de estas iniciativas y algunos factores territoriales de gran relevancia, tales como la situación socioeconómica y la dinámica colectiva de los barrios en donde surge.

\section{El mapa de innovación social de la ciudad de Valencia: contexto territorial}

Las ciudades han sido uno de los espacios más duramente golpeados por la reciente crisis económica. Pero la ciudad, por su densidad social, es también un entorno en donde la respuesta colectiva se ha podido articular de forma más efectiva, constituyendo un ámbito idóneo para el surgimiento de iniciativas de innovación social que proporcionan vías alternativas para superarla. La ciudad de Valencia es una de las metrópolis españolas que ha sufrido con más intensidad los efectos de la crisis, debido al fuerte peso que el sector inmobiliario y las actividades de menor nivel tecnológico habían adquirido en la etapa previa. A partir de 2006, la actividad económica en el área metropolitana se retrae

y, en consecuencia, el paro registrado aumenta con un ritmo superior a la media de la región. Así, entre 
2006 y 2011 , la tasa de paro estimada (paro registrado sobre población en edad de trabajar) aumentó desde el 5,6 en 2006 al 14,6\% en 2011, con un ritmo de incremento que superó la media regional (Salom y Albertos, 2014). Los efectos sociales han sido también importantes, y apenas se han reducido en los años de pretendida recuperación: en Valencia ha aumentado la pobreza (desde 2013 la presencia de familias en los comedores sociales ha aumentado más de un $60 \% 7$ ), el desempleo (la ciudad ha perdido 76.500 puestos de trabajo en los últimos 10 años $\left.{ }^{8}\right)$, y la precariedad laboral entre los grupos más vulnerables (jóvenes y mujeres ${ }^{9}$ ).

Espacialmente, los efectos han sido desiguales. Por un lado, el impacto de la crisis económica ha tendido a homogeneizar a peor la situación de los distintos municipios del área metropolitana tanto en relación con el mercado de trabajo como en cuanto a la capacidad adquisitiva y el índice de actividad económica. Por otro lado, si tenemos en cuenta la distribución de la población, los efectos negativos de la crisis se han concentrado en determinados municipios de la primera corona metropolitana, en particular los ubicados en L'Horta Nord y Sud, aumentando así las desigualdades internas medidas a través del índice de Gini (Salom y Pitarch, 2014),y agudizando así los contrastes territoriales internos (Salom y Fajardo, 2017).

En el centro urbano, la política pre-crisiscentrada en la promoción de grandes eventos yel desarrollo de megaproyectos de arquitectura de prestigio, así como los efectos diferenciados de la crisis económica, han generado un espacio urbano fuertemente fragmentado y crecientemente dualizado (Torres y García, 2013). Junto al desarrollo de nuevos espacios urbanizados que concentran la población de alta renta, los recientemente publicados resultados del estudio sobre barrios vulnerables del Ministerio de Fomento dan cuenta de una importante ampliación territorial de la vulnerabilidad en la ciudad de Valencia durante el periodo intercensal 2001-2011; mientras que en la primera fecha encontramos en la ciudad ocho barrios caracterizados como vulnerables en base a su desviación respecto a la media nacional en tasa de paro, nivel educativo y calidad urbanística; en 2011 se llegan a contabilizar hasta 32 nuevas zonas, a las que cabe sumar las ya existentes, de las cuales sólo una ha desaparecido y dos han reducido su nivel de vulnerabilidad (Ministerio de Fomento, 2016).

En este contexto, la identificación de las iniciativas de innovación social resulta de gran interés, ya que de su análisis puede surgir un conocimiento que contribuya a identificar los factores que condicionan su surgimiento, evolución y desarrollo, información útil para mejorar el diseño de políticas de apoyo específicas que contribuyan a la solución de problemas sociales relevantes. El objetivo es, pues, conocer las iniciativas de innovación social que hay en la ciudad de Valencia, la tipología de las mismas y su origen, así como comprender y analizar el escenario social concreto en el que se están desarrollando.

7.- Informe de Casa de la Caridad de 2017. Ver: http://www.levante-emv.com/valencia/2017/02/15/pobreza-cronica-valencia/1529276.html

8.- Informe de CC.OO de 2016. Ver: http://valenciaplaza.com/la-ciudad-de-valencia-ha-perdido-76500-puestos-de-trabajo-en-los-ultimos10-anos

9.- Informe del Ayuntamiento sobre la situación laboral de las mujeres, 2017. Ver: http://www.lavanguardia.com/local/valencia/20170306/42582149269/valencia-constata-en-un-informe-una-mayor-ecariedad-en-el-empleo-de-mujeres.html 


\section{Metodología de elaboración}

En el estudio se han aplicado técnicas cualitativas y cuantitativas de forma paralela, trabajando principalmente en dos líneas de investigación.

La primera de ellas se centró en el análisis del concepto de innovación, con el fin de definir criterios comunes que nos permitieran seleccionar y caracterizar las iniciativas. Las técnicas aplicadas para tal fin han sido: (1) la investigación documental, (2) cuestionario on line de consulta a los y las profesionales universitarios sobre los aspectos más importantes en innovación social, y (3) realización de un focus-group. La segunda línea de investigación se ha centrado en el análisis de caso: las iniciativas de innovación social en la ciudad de Valencia (4 y 5).

\section{(1) La investigación documental}

El análisis documental se elaboró como paso previo a la conceptualización del término innovación social; el análisis de la bibliografía no sólo permite la aproximación al significado del término, sino que también faculta al grupo de investigación para establecer límites y criterios con el fin de conocer si una iniciativa es innovadora o no, fijando la atención tanto en el objeto de la misma, como en el límite temporal en el que se desarrolla la iniciativa.

(2) Cuestionario on line sobre el concepto de Innovación Social

Para profundizar en la conceptualización, se elaboró un cuestionario on line de consulta dirigido a académicos. A lo largo del curso 2016-2017 se pidió a los profesores de la Universidad de Valencia su colaboración, en particular para acotar la definición de innovación social. Se propusieron una serie de características, a partir de la bibliografía consultada y de los resultados del focus group, con el fin de que las valorasen. Contestaron a esta encuesta 51 personas de las que sólo 4 señalan tener un conocimiento superficial del tema.

El cuestionario on line constaba de los siguientes ítems:

- Nivel de conocimiento de los expertos consultados, donde cada uno debe indicar cuál es su familiaridad con la temática abordada.

- Afirmaciones más importantes en el concepto de innovación social, donde deben indicar el nivel de acuerdo con cada una de las afirmaciones, mediante una escala Likert de 1 (menor importancia) a 5 (mayor importancia).

- Ejemplos de innovación social.

- Consulta sobre el conocimiento de iniciativas en su entorno. 


\section{(3) Focus-group}

Considerando que en el momento actual se está generando una creciente discusión acerca de la definición de Innovación Social y su alcance, se organizó un grupo focal en el que participaron seis expertos en la materia procedentes tanto del ámbito académico como del profesional. El primer taller se propuso como un espacio de intercambio de opiniones y debates en torno a dos cuestiones:

- Las características fundamentales que deben presentar las prácticas y/o iniciativas para considerarlas como innovación social.

- El papel de la Administración pública en los procesos de innovación.

(4) El estudio de casos y su clasificación

El estudio de casos ha integrado el trabajo de laboratorio y el trabajo de campo, ya que al no existir previamente un registro 0 base de datos sobre las prácticas, proyectos, iniciativas y/o agentes que trabajan directamente con la innovación social, se hizo necesario partir de la consulta de las convocatorias de distintos tipos de ayudas a proyectos de innovación social (convocatorias del Ayuntamiento de Valencia de los ejercicios 2015 y 2016 ${ }^{10}$, convocatorias de ayudas Emergents impulsadas por el Vicerrectorado de Cultura e Igualdad de la Universitat de València de estos mismos años ${ }^{11}$ ), así como el rastreo a través de redes sociales, web y diferentes fuentes documentales. Esta indagación inicial permitió registrar un total de 112 iniciativas, que tras un proceso de depuración fueron reducidas a 79 iniciativas que a priori trabajaban con y para la innovación social en la ciudad de Valencia.

Estas iniciativas fueron clasificadas en siete categorías cuyo principal criterio fue el objeto de la innovación social por el que se ha reconocido la iniciativa:

- Ecología urbana y consumo (12 iniciativas)

- Dinámicas sociales e inclusión (19 iniciativas),

- Urbanismo y Patrimonio (10 iniciativas)

- Estructuras de apoyo al emprendedurismo (9 iniciativas)

- Redes de intercambio y economía colaborativa:(15 iniciativas)

- Fomento del empleo y orientación profesional: (6 iniciativas)

- Educación (8 iniciativas)

10.- El objeto principal de estas ayudas es apoyar la realización de proyectos de innovación social siempre y cuando estén orientados a la resolución de problemas sociales que afectan al municipio de Valencia.

11.- Estas ayudas pretenden ser un impulso para el desarrollo de proyectos de creatividad, innovación e inclusión sociocultural, destinados a promover la inclusión social y cultural en el término municipal de Valencia, premiando aquellas propuestas que desencadenen cambios innovadores hacia una mayor justicia social. 
(5) La representación gráfica: características técnicas del mapa

La representación gráfica de las iniciativas identificadas se integró inicialmente ydurante el proceso de trabajo en una herramienta gratuita online, "MyMaps", una plataforma online que ofrece "Google". Este tipo de herramienta permite que el mapa pueda ser editado y actualizado de manera constante, así como una personalización de los iconos que caracterizan el tipo de iniciativa. Además, su simplicidad permite la consulta por parte de las entidades e iniciativas contactadas, facilitando su grado de respuesta.

\section{Resultados}

\subsection{Aportaciones a la definición y uso del concepto: Focus Group y encuestas}

La innovación social es un término que tienen muchas y variadas interpretaciones. Los expertos participantes en el focus-group señalaron que es preferible hablar de innovación sociocultural, porque no hay ni un solo proceso social que no sea al mismo tiempo cultural, y en ello parecen estar de acuerdo todos los participantes. Debe existir un cambio cultural para que se produzca un cambio en la sociedad, es decir, un cambio en las mentalidades. Si no hay cambio cultural, difícilmente podrá haber innovación social. El concepto de creatividad es importante en la innovación social, y debe estar presente en su definición, ya que comporta los siguientes elementos:

a) Originalidad, es decir, no reproducimos lo que ya existía, debe haber novedad en el proceso. El uso del "concepto original y novedoso" abre debate durante el intercambio de opiniones entre los expertos. Por un lado, encontramos las opiniones que se basan en la definición de innovación social según la Stanford Social Innovation, y, por otro, las opiniones que se apoyan en la definición de María Elisa Bernal, basada en las experiencias de la CEPAL ${ }^{12}$. Esta última plantea que la innovación social son nuevas formas de hacer las tareas tradicionales, y que esas tareas deben ser replicables en distintos contextos, por lo que la novedad no es tan importante, como sí lo es para los expertos de la Universidad de Stanford. En lo que todos coinciden es en la idea de que la relación coste-beneficio debe ser mejor que la de los modelos tradicionales, es decir, debe ser más eficaz, eficiente y sostenible que las soluciones actuales. 
b) Adecuación, es decir, los procesos que introducen las innovaciones son adecuados para el problema social que quieren resolver o paliar, hay innovación automática. Además, la innovación social apuesta por procesos colectivos y los antepone a los intereses individuales. La innovación estimula el desarrollo de competencias a partir de la comunicación, interacción y participación comunitaria.

c) Orientada al futuro, ya que en el mundo actual nos movemos en escenarios de inseguridad e incertidumbre, y en esos escenarios los procesos de acción colectiva trata de establecer límites y de encontrar como gestionar esas incertidumbres. La innovación no sólo debe ser replicable y adaptable a distintas circunstancias, sino también resiliente, debe ser capaz de resolver distintos problemas, dudas que puedan surgir en determinados contextos.

d) Capacidad de resolver problemas sociales, es decir, tiene una aportación en clave positiva y no negativa para la sociedad. Con frecuencia no distinguimos entre bienes personales, bienes cívicos, bienes comunales y bienes públicos. Surge como consecuencia de un fallo: en el mercado, gobierno y/o sector del voluntariado, lo cual explica, y obliga, en cierta manera, a que surjan agentes con una misión clara, la de generar un valor social. El objetivo de la innovación social es crear cambio social y regenerar la sociedad. La lucha contra la desigualdad está entre los objetivos básicos de cualquier iniciativa de innovación social.

A modo de conclusión, y para poder determinar las características fundamentales que deben presentar las prácticas y/o iniciativas para considerarlas como innovación social, los elementos fundamentales que debe tener dicha definición, en los que coinciden los expertos, son:

- La innovación social surge como consecuencia de un fallo, ya sea fallo de mercado, de gobierno, etc.

- Debe crear un cambio social y regenerar la sociedad, dando soluciones creativas a problemas sociales, y

- Debe ser entendida como un proceso colectivo que genere un impacto social positivo.

La Administración actúa como fuente de diferentes procesos de Innovación Social. Pero no es menos cierto que existen estructuras administrativas que muchas veces inhiben la toma de iniciativas en temas de innovación, y que todavía continúan promoviendo respuestas estandarizadas y unidireccionales a problemas complejos y multidimensionales. Existe una carencia de diálogo, de colaboración, sinergia y cohesión entre los diferentes agentes promotores de Innovación Social. Esto no significa que la Administración pública no impulse los procesos de Innovación Social, sino que la innovación en la esfera pública está demasiado reducida a ciertos entornos y limitada por su propia estructura económica, organizativa y relacional.

En segundo lugar, los resultados de las encuestas a profesores universitarios nos ayudan a centrar el tema y nos despiertan nuevas reflexiones. La mayoría de los que han respondido a la encuesta conocen el término innovación social (76\%), aunque el 39\% sólo de manera superficial. El $6 \%$ se consideran a sí mismos expertos, el $28 \%$ lo conocen como parte de su ámbito de estudio y el $27 \%$ lo cono- 
cen por interés cultural. De todos los centros de la universidad, ha sido la facultad de Economía el que ha dado más respuestas, seguido por la facultad de Ciencias Sociales, Geografía e Historia y Medicina. El resto de centros han participado de manera minoritaria.

Los aspectos que se han tratado, y valorado, en la encuesta son los siguientes:

- La innovación social es una nueva forma de satisfacer las necesidades sociales.

- La innovación social trata de resolver los retos sociales, culturales, económicos y/o ambientales.

- La innovación social responde a un reto social no cubierto.

- Una innovación social tiene que ser una idea nueva en el entorno en que se aplica.

- Una innovación social puede ser una nueva forma de aplicar una vieja idea.

- La innovación social propone soluciones cuyo valor prima al bien común de la sociedad por encima del individual.

- La innovación social se lleva a cabo contando con la participación de la comunidad.

- Sólo hay innovación social cuando la idea se lleva a la práctica.

- La innovación social debe ser reproducible en distintos contextos.

- La innovación social crea nuevos vínculos, nuevas relaciones sociales y de colaboración.

- La innovación social debe impactar fuertemente a nivel local.

- La innovación social favorece el sentimiento de ciudadanía.

Cabe destacar la gran importancia que los expertos (aquellos que se han calificado a sí mismos como expertos) dan a aspectos relacionados con las transformaciones sociales y la aplicación práctica para definir la innovación social. Los que conocen el tema pero no se consideran expertos, sin embargo, indican que características como la novedad, la aplicación práctica y la participación resultan de gran importancia en la definición. Para los menos, conocen la innovación social por cultura general o de manera superficial, señalan que la innovación está muy relacionada con la consolidación de relaciones sociales, pero que la replicabilidad no tiene demasiada importancia.

Las diferentes especialidades de los participantes en la encuesta también parece que influyen en su concepción de la innovación social. Mientras que los economistas y los sociólogos dan mucha importancia a los retos sociales (los primeros en particular a los retos no cubiertos), los profesores de Geografía e Historia, así como de Medicina consideran relevantes los aspectos relacionados con el desarrollo comunitario, sin embrago, los primeros no dan ninguna importancia a la puesta en práctica de la idea, siendo para los médicos uno de los aspectos más significativos. El resto de especialidades dan gran importancia a la generación de valor que prima al bien común de la sociedad por encima del individual. Los economistas inciden en la novedad, mientras que no ocurre así entre los sociólogos.

Ante la pregunta sobre la calificación como innovación social de distintas iniciativas, la mayor parte de los encuestados identifican mayoritariamente los bancos de tiempo (35 respuestas), los espacios de co-working (33 respuestas), los huertos urbanos (29 respuestas), las incubadoras de empresas (29 
respuestas) y las monedas sociales (28 respuestas) como las principales innovaciones sociales. Entre los científicos sociales, las iniciativas ligadas a los bancos de tiempo destacan ligeramente, mientras que entre economistas y médicos lo hacen las incubadoras de empresas sociales. Entre los profesores de Geografía e Historia destaca, también ligeramente, los espacios de co-working.

Como conclusión, la definición propuesta en nuestro análisis de la innovación social es la siguiente: Una innovación social es una idea, o una nueva forma de aplicar una vieja idea. Se trata de hacer las cosas de manera diferente. Aparece cuando la sociedad re-examina cómo se pueden hacerse las cosas. Debe ser novedosa en el contexto en que se inserta (no es necesario que sea una innovación absoluta), resuelve retos sociales, culturales, económicos y/o ambientales, puede ser llevada a cabo por el sector público, privado, o tercer sector, puede ser endógena (surgir de las personas que la necesitan) o exógena (de personas que quieren ayudar), se orienta para el beneficio común de la población, se prima el bien común de la sociedad antes que beneficios individuales, se realiza contando con la participación de la comunidad y los beneficiarios, que se transforman en actores de su propio desarrollo, fortaleciendo así el sentimiento de ciudadanía -a participación de los agentes locales supone el empoderamiento y el compromiso del ciudadano-, tiene que haber sido llevada a la práctica (no sólo una idea), y ser reproducible, debe ser más productiva, efectiva, eficiente y/o sustentable que soluciones previas, debe ser capaz de crear nuevos vínculos, nuevas relaciones sociales y de colaboración y debe impactar fuertemente -y de manera positiva en cuanto a los objetivos del desarrollo sostenible- a nivel local.

\subsection{Tipología de las iniciativas detectadas}

Las 79 iniciativas identificadas (figura 1) se han agrupado en siete categorías ordenadas teniendo en cuenta el objeto de la innovación de cada una de ellas, siendo la categoría Dinámicas sociales e inclusión la que agrupa un mayor número de iniciativas que suponen el $24 \%$ del total, seguida de las iniciativas unificadas en la categoría de Redes de intercambio y economía colaborativa con un diecinueve por ciento. Un poco por debajo se encuentra Ecología urbana y consumo que supone un 15\%, y Urbanismo y patrimonio con un $13 \%$. Las categorías con un porcentaje inferior son las Estructuras de apoyo al emprendedor que suponen un 11\% por ciento, Educación con un diez por ciento y Fomento del empleo y orientación profesional con un $9 \%$ de las iniciativas que han sido identificadas.

Las iniciativas promovidas por el Tercer Sector (figura 2) aparecen en todas las categorías, aunque de manera más destacada lo hacen en las agrupaciones de Ecología urbana y consumo, Dinámicas sociales e inclusión, y Redes de intercambio y económica colaborativa, en las que encontramos 5 iniciativas en cada una de ellas. Es pertinente resaltar que las iniciativas en este sector crecen de manera progresiva, pero tienen un repunte significativo en los años 2013, 2014 y 2015, el cual no podemos desvincular del contexto socio político del momento, en el que las partidas presupuestarias para la financiación de las organizaciones no gubernamentales sufrieron un recorte significativo, al tiempo que abrían las primeras convocatorias de financiación dirigidas a la innovación social, 
que supuso para algunas entidades una oportunidad de redefinir sus actividades y promocionarse por esta vía. El sector público es el que menor representatividad tiene con solo tres iniciativas en el periodo más reciente.

\section{Figura 1. Clasificación de las iniciativas de Innovación Social (\%)}

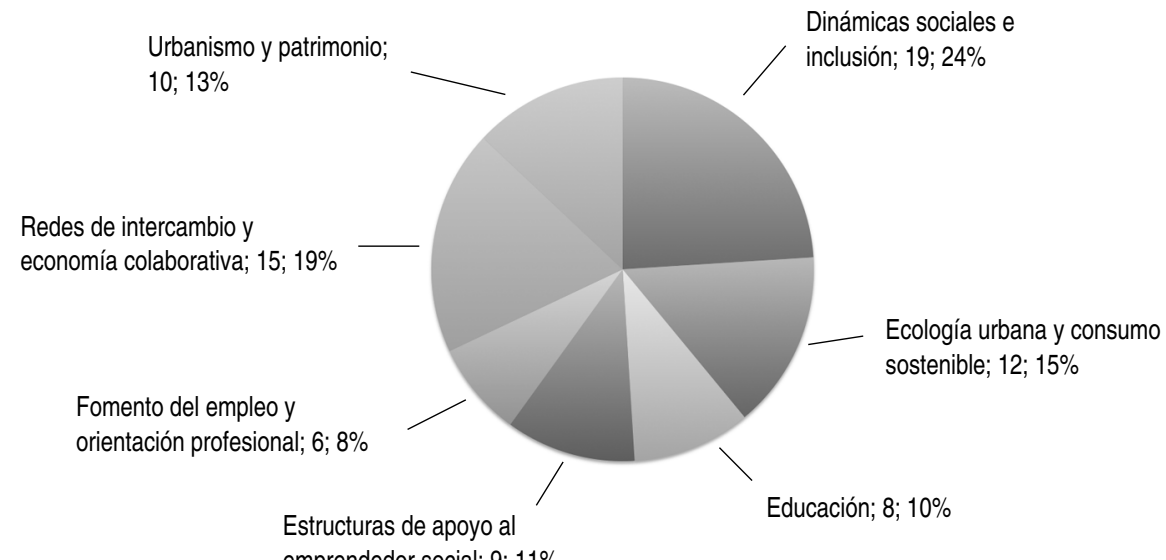

\section{Figura 2. Tipología de las iniciativas de Innovación Social y año de aparición (2010-2017)}

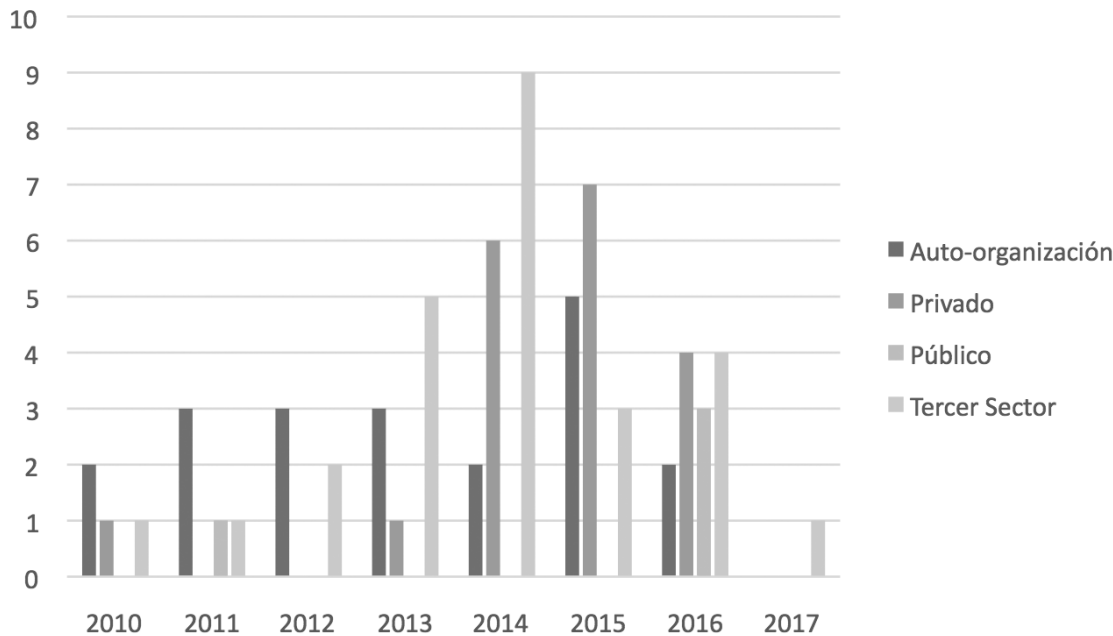

CIRIEC-España, Revista de Economía Pública, Social y Cooperativa 


\subsection{Ubicación geográfica y relación con los barrios}

Nuestra hipótesis de partida es que el surgimiento de las iniciativas de innovación social está estrechamente relacionado con las condiciones geográficas (sociodemográficas, económicas y territoriales) del entorno en que aparecen. Como puede verse en la figura 2, las iniciativas innovadoras identificadas se concentran geográficamente en un número limitado de barrios y distritos de la ciudad. Los distritos de Ciutat Vella y Benimaclet son los que concentran un mayor número de iniciativas (15 y 10 respectivamente). Junto con Extramurs,Algirós, y Poblats Marítims ( 8,6 y 5 ) iniciativas respectivamente), suman el $66 \%$ del total. Son estos mismos distritos los que concentran el mayor número en términos relativos a la población que reside en ellos y a la superficie que abarcan. En relación con la población, destacan especialmente los distritos de Ciutat Vella(5,6iniciativas por cada 10.000 habitantes) y Benimaclet $(3,4)$. Los únicos distritos en donde no se ha detectado ninguna iniciativa son $\mathrm{El}$ Plá del Real, Poblats del Nord y Poblats del Oest.

\section{Figura 3. Localización de las iniciativas de innovación social (2016-17) y densidad de iniciativas en función de la población por distritos}

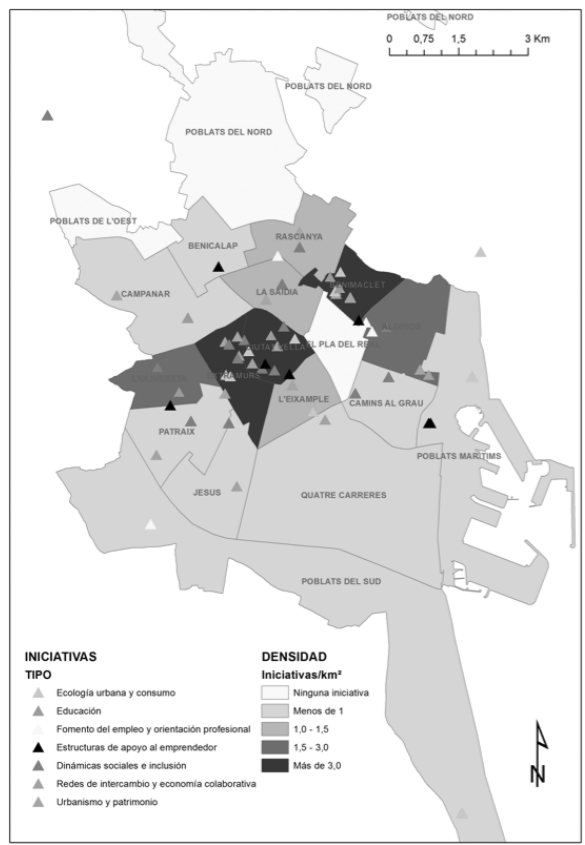

FUENTE: Mapa de innovación social de la ciudad de Valencia elaborado por la Cátedra Ciudad de Valencia y Padrón de Población 2016. 
Un examen más detallado nos permite observar la relación que mantienen los procesos estudiados con determinados barrios. Existe una concentración relativa de iniciativas en determinadas zonas de la ciudad, que agrupan barrios contiguos, en muchos casos de distintos distritos municipales. Las tres áreas principales son las siguientes:

- La primera está constituida por los distintos barrios de Ciutat Vella, en donde, salvo en el caso de El Mercat, encontramos los índices más elevados de iniciativas en relación con la población (más de 13 iniciativas por cada 10.000 habs. en La Seu, 11 en Sant Francesc, 5 y 4 respectivamente en la Xerea y el Pilar), a los que podemos añadir el contiguo barrio de El Botànic, también con un índice por habitante elevado $(7,6)$;

- En segundo lugar, la zona constituida por el distrito de Benimaclet, La Carrasca y la Vega Baixa en el distrito de Algirós, y Els Orriols y Torrefiel en Rascanya.

- En tercer lugar, Els Poblats Marítims, en particular el barrio de El Grau, aunque también pueden incluirse los vecinos barrios de Cabanyal-Canyamelar y Beteró, con menor número de iniciativas pero que suponen un índice relativamente elevado en relación con su población residente.

Las dos primeras destacan por tener presencia de iniciativas en todas las categorías analizadas, aunque con un mayor peso de los proyectos de educación e intercambio y economía colaborativa en el caso de Benimaclet-Els Orriols, y de educación e inclusión en Ciutat Vella. En el caso de Els Poblats Marítims, las iniciativas se concentran en el ámbito de la ecología urbana y consumo, y la presencia de estructuras de apoyo al emprendedor, no habiéndose identificado iniciativas relacionadas con la educación, el empleo y las redes de intercambio.

Aunque es evidente que nos encontramos ante procesos sociales complejos en los que intervienen distintas variables, y cuya interpretación no puede realizarse más que a partir de un pormenorizado análisis cualitativo, todavía en desarrollo, sí es posible avanzar algunas hipótesis que explican las causas de esta localización.

En primer lugar, la existencia de iniciativas parece estar relacionada con el nivel de renta de la población. La literatura sobre innovación social considera que ésta surge como un medio para luchar contra la exclusión social y mejorar la calidad de vida; de forma que los mecanismos de crisis y recuperación provocany aceleran la innovación social (Moulaert et al., 2010). De esta forma, la mayoría de las iniciativas de innovación social surgirían como reacción a condiciones de exclusión social o a una baja calidad de vida. Esta vinculación explicaría la ausencia de este tipo de proyectos en el distrito de El Plá del Real, y su escasa frecuencia en Penya-Roja y los distintos barrios de L'Eixample, habitados por población con un nivel de renta elevado y en ausencia de conflictos relacionados con la multiculturalidad. Sólo en el barrio de Sant Francesc encontramos coincidencia entre una buena situación socioeconómica y alta densidad de iniciativas, que puede atribuirse a su papel como CBD urbano y su consiguiente capacidad de atracción para la ubicación de todo tipo de establecimientos y servicios. 
No obstante, la variable renta es una explicación insuficiente, ya que también encontramos escasez de iniciativas en distritos como Quatre Carreres, Jesús, Pobles del Oest y Pobles del Nord, con un nivel de renta mucho más bajo y un mayor porcentaje de población inmigrante. Por otra parte, los núcleos más activos de innovación social no se corresponden necesariamente con la peor situación: Tanto Benimaclet, Algirós o la Carrasca como los barrios de Ciutat Vella y Extramurs con mayor densidad de iniciativas, tienen niveles educativos medios, así como niveles medio-altos de inmigración.Sólo en el caso de Els Poblats Marítims coincide la existencia de población con bajo nivel socioeconómico con una alta densidad de iniciativas. Estos resultados no concluyentes son similares a los obtenidos en un estudio similar realizado en Cataluña (Blanco et al., 2013). En aquel caso, los autores concluyen que la innovación social no surge necesariamente en las comunidades más necesitadas, en donde los factores de exclusión social tienden a concentrarse, sino en zonas que concentran más recursos para la acción colectiva.

En consecuencia, hemos apuntado como una segunda variable explicativa la vinculación de una parte de estas iniciativas con los movimientos sociales y ciudadanos. Según Martinelli (2010), la innovación social en comunidades urbanas tiene una estrecha relación con los movimientos sociales; y la innovación social ocurre cuando la acción colectiva se dirige a conseguir cambios en relación con la satisfacción de necesidades humanas no consideradas o satisfechas, el empoderamiento de grupos sociales marginalizados a través de la ampliación de las capacidades y la (re)creación de identidad, y las relaciones sociales, de poder y/o gobernanza dentro de la comunidad y entre la comunidad y el conjunto de la sociedad.

Esta hipótesis se sustenta en nuestro caso en la constatación de que una parte importante de las iniciativas identificadas guarda relación con el movimiento de los "indignados", que tuvo una especial incidencia en la ciudad de Valencia. Así, un número elevado de las iniciativas (el $65 \%$ de las detectadas) surge en los años 2014-2016 y, tal y como se ha constatado en las entrevistas, se encuentran vinculadas de algún modo a los participantes en este movimiento. Para verificarlo, hemos contrastado la localización de las iniciativas con el porcentaje del voto en las elecciones municipales de 2015 que se dirigió a los partidos relacionados más estrechamente con este movimiento, y que fueron los principales artífices del cambio de gobierno municipal en la ciudad: "Valencia en Comú", "Compromís" y "EUPV-EV-ERPV-AS:AC"13.

Pero quizás la variable más importante, y sobre la que será necesario profundizar a partir de análisis cualitativos, está relacionada con el tejido social y asociativo, las redes y el capital social consolidado a lo largo del tiempo. Así, los tres núcleos de innovación principales que hemos identificado coinciden con zonas con una larga tradición de sociabilidad formal o informal; se trata de barrios que han constituido tradicionalmente importantes focos de asociacionismo, vinculados a un potente movi- 
miento vecinal, a un entramado festivo excepcionalmente denso, y a distintos tipos de asociaciones recreativas o culturales (Torres y García, 2013). Estas redes sociales se han constituido en respuesta a una situación de marginación prolongada en el tiempo y a los conflictos territoriales y sociales que les han afectado. Los barrios que actualmente presentan una mayor intensidad de iniciativas arrastran una larga historia de vulnerabilidad social; Cabanyal-Canyamelar-Beteró era ya considerada un área vulnerable en el mapa de Fomento de 1991; mientras que Carme-Mercat-Velluters y Els Orriols, así como los barrios de Tres Forques-La Fontsanta o Sant Antoni-Tormos-Morvedre, forman parte de este mapa desde 2001.

Se trata, además, de barrios afectados por proyectos urbanísticos que han generado controversia y contestación, lo que ha dado lugar a movimientos sociales de protesta que han generado una tradición de cooperación en un contexto de búsqueda de soluciones alternativas. Destaquemos, entre otros, el conflicto derivado del proyecto urbanístico del denominado sector Este de Benimaclet, que llevó a los vecinos a ocupar las tierras destinadas a este proyecto para instalar huertos urbanos comunitarios (Del Romero, 2014), los irregulares resultados de la política de renovación urbana en Ciutat Vella (Hernández y Torres, 2013), o el proyecto de prolongación de la avenida Blasco Ibáñez, que supuso la consolidación de la plataforma "Salvem el Cabanyal" (Ruíz y García, 2013).

Finalmente, no debemos dejar de mencionar como un factor a considerar la llegada reciente a Ciutat Vella y Benimaclet de nuevos vecinos, tanto población inmigrantecomo estudiantes y jóvenes profesionales con alto nivel educativo, que contribuyen a constituir espacios rejuvenecidos, multiculturales y dinámicos en principio propicios al surgimiento de tales iniciativas.

\section{Conclusiones}

En definitiva, la delimitación del término "innovación social" es compleja y supone un posicionamiento epistemológico y también político. Sin embargo, dado el interés del término y su cada vez mayor presencia en la agenda política, es importante saber de qué tipo de iniciativas y empresas estamos hablando porque ello tendrá repercusión clara en la práctica de la administración pública (desarrollo de normativa, subvenciones, etc.). La Unión Europea y los gobiernos locales parecen tener especial interés en este tipo de fenómenos cuyo objetivo, resumiendo, es dar solución a problemas sociales a los que ni el Estado ni el mercado han sabido responder adecuadamente. Es cierto que se trata de iniciativas, que muchas veces no pueden calificarse como empresas, que surgen en los márgenes del mercado, como acciones espontáneas basadas en la colaboración altruista de sus socios o de las personas interesadas. Su objetivo suele solucionar un problema, pero no sólo problemas relacionados 
con la subsistencia, como la pobreza o la desigualdad, temas de enorme trascendencia social e histórica, sino también asuntos que afectan a la forma de vida ligada al sistema capitalista imperante y contra los cuales una parte de la población se revela porque no identifica moral ni éticamente con los resultados que ello acarrea. Esto resulta más importante que lo anterior, sobre todo en determinados entornos o contextos como son las ciudades del mundo occidental, en particular las europeas, y la clase media que, por una parte ha visto, con la crisis, mermada su capacidad de acción, en especial política, pero que, por otra parte, sin haber entrado en procesos de precarización económica, reclaman una forma diferente de consumo, de participación democrática y de entorno social y natural.

La cuantificación y caracterización de estas iniciativas de innovación social a nivel local es una forma de conocerlas, en muchas ocasiones su visibilización es escasa o nula, y contribuir a su conocimiento por parte de la sociedad a la que, en última instancia, tratan de transformar. En el caso de la ciudad de Valencia, la Cátedra Ciudad, financiada por el Ayuntamiento, ha elaborado un mapa de la innovación social dando la posibilidad a estas iniciativas de ser conocidas por los ciudadanos y también por la administración local. El proyecto se encuentra aún en proceso de elaboración y el objetivo es que se convierta en una plataforma abierta de intercambio de ideas y procesos innnovadores que contribuyan a mejorar la calidad de vida en la ciudad.

\section{Bibliografía}

BLANCO, I., CRUZ, H., MARTÍNEZ, R. \& PARES, M. (2016): "El papel de la innovación social frente a la crisis", Ciudad y territorio. Estudios Territoriales, XLVIII (188), 249-260.

CAULIER-GRICE, J., DAVIES, A., PATRICK, R., \& NORMAN, W. (2012): Social innovation overview: a deliverable of the project: "The theoretical, empirical and policy foundations for building social innovation in Europe" (TEPSIE), European Commission - 7th framework programme, DG Research, Brussels.

CHOI, N. \& MAJUMDAR, S. (2015): "Social innovation: towards a conceptualisation". In: Majumdar, S., Guha, S., Marakkath, N. (Eds.), Technology and innovation for social change, Springer India, 7-34.

CONEJERO, E. \& REDONDO, J.C. (2016): "La innovación social desde el ámbito público: conceptos, experiencias y obstáculos", Gestión y Análisis de Políticas Públicas: Nueva época, 15, 1-20.

DEL ROMERO, LI. (2014): "Movimientos sociales y conflictos territoriales: el caso de Barcelona y Valencia", Cuadernos de Geografía, 94, 17-35. 
DEUSTO INNOVACIÓN SOCIAL (Julio de 2016): Deusto Innovación Social. Nuevos valores y actitudes en un mundo en transformación, Universidad de Deusto, Obtenido de: https://blogs.deusto.es/innovacionsocial/que-es-deusto-innovacion-social/

EUROPEAN COMMISSION (2013): Guide to Social Innovation.Bruselas, European Comission.

GARCÍA, M., EIZAGUIRRE, S. \& PRADEL, M. (2015): "Social innovation and creativity in cities: A socially inclusive governance approach in two peripheral spaces of Barcelona", City, Culture and Society, 6, 93-100.

GEROMETTA, J., HAUSSERMANN, H. \& LONGO, G. (2005): "Social Innovation and Civil Society in Urban Governance: Strategies for an Inclusive City", Urban Studies, 42 (11), 2007-2021.

HEISKALA, R. (2007): "Social innovations: structural and power perspectives", Social innovations, institutional change and economic performance, Edward Elgar, Cheltenham, 52-79.

HERNÁNDEZ, G.M. \& TORRES, F. (2013): "El impacto de la Valencia glocalizada en el Centro Histórico popular". En: Cucó i Giner, J. (dir.), La ciudad pervertida. Una mirada sobre la Valencia global, Barcelona, Antrhopos, 19-40.

HERNÁNDEZ-ASCANIO, J., TIRADO-VALENCIA, P. \& ARIZA-MONTES, A. (2016): "El concepto de innovación social: ámbitos, definiciones y alcances teóricos", CIRIEC-España, Revista de Economía Pública, Social y Cooperativa, 88, 165-199.

Jornada sobre Innovación social. Logroño, 13 de diciembre de 2012 (Julio de 2016). Obtenido de Instituto Nacional de Administración Pública, http://www.inap.es/

LÓPEZ, C., PRIEDE, T. \& DEL ARCO, J. (2015): "La empresa social. Experiencias innovadoras a través del deporte", REVESCO, Revista de Estudios cooperativos, 119, 107.

MARCY, R. \& MUMFORD, M. (2007): "Social Innovation: Enhancing Creative Performance Through Causal Analysis", Creativity Research Journal, 19(2), 123-140.

MARTINELLI, F. (2010): "Historical roots of social change. Philosophies and movements". In: Moulaert, F., Martinelli, F., Swyngedouw, E. \& González, S., Can Neighbourhoods Save the City?, London and New York, Routledge, 17-48.

MARTíNEZ, R. \& SUBIRATS, J. (2015): Innovación social: ¿Más mercado o más sociedad?, Barcelona, IGOP.

MÉNDEZ, R. (2015): "Redes de colaboración y economía alternativa para la resiliencia urbana: una agenda de investigación", Biblio 3W, 20, 1-24.

MÉNDEZ, R. (2016): "Los nuevos escenarios económicos: innovación en los espacios urbanos". VII Jornadas de Geografía Económica, Los escenarios económicos en transformación. La realidad territorial tras la crisis económica, Universidade de Santiago de Compostela-Grupo de Geografía Económica de la AGE. Celebrado entre los días 6-8 julio. 
MINISTERIO DE FOMENTO (2016): Visor del catálogo de barrios vulnerables, http://www.fomento.gob.es/MFOM.BarriosVulnerables/, 9/5/2017, Madrid.

MORALES, A.C. (2010): "Claves para comprender la innovación social”, Social Innova, 13-40.

MORALES, A.C. (2010): "Tercer sector e innovación: experiencias, desafíos y oportunidades", Revista Internacional de Organizaciones, 5, 165-183.

MOULAERT, F. (2009): "Social innovation: Institutionally embedded, territorially (re) produced", Social innovation and territorial development, 11-24.

MOULAERT, F. (Ed.). (2013): The international handbook on social innovation: collective action, social learning and transdisciplinary research, Edward Elgar Publishing.

MOULAERT, F., MACCALLUM, D. \& HILLIER, J. (2013): "Social innovation: intuition, precept, concept", The International Handbook on Social Innovation: collective action, social learning and transdisciplinary research, 13-39.

MOULAERT, F., MARTINELLI, F., GONZÁLEZ, S. \& SWYNGEDOUW, E. (2007): "Introduction: Social innovation and governance in European cities urban development between path dependency and radical innovation", European Urban and Regional Studies, 14(3), 195-209.

MOULAERT, F., MARTINELLI, F., SWYNGEDOUW, E. \& GONZÁLEZ, S. (2005): "Towards alternative model (s) of local innovation", Urban studies, 42(11), 1969-1990.

MOULAERT, F., MARTINELLI, F., SWYNGEDOUW, E. \& GONZÁLEZ, S. (2010): Can Neighbourhoods Save the City?, London and New York, Routledge.

MULGAN, G., TUCKER, S., ALI, R. \& SANDERS, B. (2007): Social Innovation. What it is, why it matters and how it can be accelerated, Skoll Centre for Social Entrepreneurship. Oxford Said Business School. The Young Foundation.

MURRAY, R., CAULIER-GRICE, J. \& MULGAN, G. (2010): The open book of Social Innovation, NESTA, Innovating public services, The Young Foundation.

OCDE (2010): SMEs, Social Entrepreneuship and Social Innovation. En: http://dx.doi.org/10.1787/9789264080355-en

OECD/European Communities (2005): Oslo Manual: Guideliness for Collecting and Interpreting Innovation Data, 3d. ed., OECD/EC, TIC y Discapacidad (2007), Madrid, Fundación Vodafone.

PERAZA CASTANEDA, E.H., GÓMEZ GARCÍA, J.M. \& ALEIXANDRE MENDIZÁBAL, G. (2016): "Los factores determinantes del comportamiento innovador de las cooperativas: un análisis para el caso de Castilla y León", REVESCO, Revista de Estudios Cooperativos, 122.

PHILLS, J.A., DEIGLMEIER, K. \& MILLER, D.T. (2008): "Rediscovering social innovation", Stanford Social Innovation Review, 6(4), 34-43, https://ssir.org/articles/entry/rediscovering_social_innovation (consultado 1 de abril de 2017). 
PITARCH, M.D., SALOM, J. \& FAJARDO, F. (2016): "Nuevos y viejos espacios-problema tras la crisis. Impacto de la política de provisión de servicios públicos de proximidad en la ciudad de Valencia", XIII Congreso de Geografía Urbana, Girona.

REGLAMENTO (UE) No 1296/2013 DEL PARLAMENTO EUROPEO Y DEL CONSEJO de 11 de diciembre de 2013 relativo a un Programa de la Unión Europea para el Empleo y la Innovación Social («EaSl») y por el que se modifica la Decisión no 283/2010/UE, por la que se establece un instrumento europeo de microfinanciación para el empleo y la inclusión social. http://eurlex.europa.eu/LexUriServ/LexUriServ.do?uri=OJ:L:2013:347:0238:0252:Es:PDF (consultado 10 de abril de 2017).

RODRÍGUEZ-HERRERA, A. \& ALVARADO, H. (2008): Claves de la innovación social en América Latina y el Caribe, CEPAL.

RUIZ, M.A. \& GARCÍA, P. (2013): "Disolución del lugar y espacios del miedo en el Cabanyal", En: Cucó i Giner, J. (dir.), La ciudad pervertida. Una mirada sobre la Valencia global, Barcelona, Anthropos, 67-92.

SALOM CARRASCO, J. \& ALBERTOS, J.M. (2006): "Valencia industrial districts facing the economic crisis: Is reinsdustrialization possible?". In: Salom, J. \& Farinós, J. (eds.), Identity and Territorial Character. Re-Interpreting Local-Spatial Development, Valencia, PUV-Publicaciones de la Universidad de Valencia, 49-80.

SALOM, J. \& PITARCH, M.D. (2014): "El área metropolitana de Valencia en la crisis". En: Albertos, J.M. y Sánchez, J.L. (coords.), Geografía de la crisis económica en España, Valencia, PUVPublicaciones de la Universidad de Valencia, 539-563.

SALOM, J. \& FAJARDO, F. (2017): "Cambios recientes en la estructura territorial sociodemográfica del Área Metropolitana de Valencia (2001-2011)", Boletín de la Asociación de Geógrafos Españoles, 73, 123-147

SZYMANSKA, A. \& JEGERS, M. (2016): "Modelling social enterprises", Annals of Public and Cooperative Economics, 87, 501-527.

THE CENTRE FOR SOCIAL INNOVATION (2011): PROOF: How shared spaces are changing the world, The Centre for Social Innovation, Toronto, Creative Commons Attribution.

TORRES, F. \& GARCÍA, P. (2013): "La ciudad fragmentada. Análisis comparativo de cuatro barrrios emblemáticos". En: Cucó i Giner, J. (dir.), La ciudad pervertida. Una mirada sobre la Valencia global, Anthropos, Barcelona, 191-212. 


\section{AGRADECIMIENTOS}

Este artículo se ha elaborado con el apoyo de la Cátedra Ciudad de la Universidad de Valencia, iniciativa financiada por el Ayuntamiento de Valencia a través de la Fundación "Las Naves", y en el marco del proyecto "Sostenibilidad social, conectividad global y economía creativa como estrategias de desarrollo en el Área metropolitana de Valencia" (CSO2016-74888-C4-1-R), financiado por la Agencia Estatal de Investigación (AEI) y el Fondo Europeo de Desarrollo Regional (FEDER) dentro del Programa Estatal de Investigación, Desarrollo e Innovación Orientada a los Retos de la Sociedad, en el marco del Plan Estatal de Investigación Científica y Técnica y de Innovación 2013-2016, convocatoria de 2016.

Deseamos agradecer a todos los que han participado de manera altruista en la metodología cualitativa, en particular a los integrantes del Focus-Group: Antonio Ariño, vicerrector de Cultura e Igualdad y profesor de Sociología, Universidad de Valencia; Rafael Chaves Ávila, profesor de Economía Aplicada y especialista en Economía social y cooperativa, Universidad de Valencia; Ester Alba, decana de la Facultad de Geografía e Historia y profesora de Historia del Arte, Universidad de Valencia; Amparo Cervera, profesora de Comercialización e Investigación de Mercados y Directora del Instituto Universitario de Economía Internacional, Universidad de Valencia; Raúl Contreras, emprendedor social y técnico de la fundación Las Naves, y Lourdes Mirón, presidenta de Jovesolides. 